\title{
ARTICLE \\ Benzoylaconine induces mitochondrial biogenesis in mice via activating AMPK signaling cascade
}

\author{
Xiao-hong Deng ${ }^{1,2}$, Jing-jing Liu ${ }^{1,2}$, Xian-jun Sun $^{1,2}$, Jing-cheng Dong ${ }^{1,2}$ and Jian-hua Huang ${ }^{1,2}$
}

\begin{abstract}
The traditional Chinese medicine "Fuzi" (Aconiti Lateralis Radix Praeparata) and its three representative alkaloids, aconitine (AC), benzoylaconine (BAC), and aconine, have been shown to increase mitochondrial mass. Whether Fuzi has effect on mitochondrial biogenesis and the underlying mechanisms remain unclear. In the present study, we focused on the effect of BAC on mitochondrial biogenesis and the underlying mechanisms. We demonstrated that Fuzi extract and its three components $A C, B A C$, and aconine at a concentration of $50 \mu \mathrm{M}$ significantly increased mitochondrial mass in HepG2 cells. BAC $(25,50,75 \mu \mathrm{M})$ dose-dependently promoted mitochondrial mass, mtDNA copy number, cellular ATP production, and the expression of proteins related to the oxidative phosphorylation (OXPHOS) complexes in HepG2 cells. Moreover, BAC dose-dependently increased the expression of proteins involved in AMPK signaling cascade; blocking AMPK signaling abolished BAC-induced mitochondrial biogenesis. We further revealed that BAC treatment increased the cell viability but not the cell proliferation in HepG 2 cells. These in vitro results were verified in mice treated with BAC $(10 \mathrm{mg} / \mathrm{kg}$ per day, ip) for 7 days. We showed that BAC administration increased oxygen consumption rate in mice, but had no significant effect on intrascapular temperature. Meanwhile, BAC administration increased mtDNA copy number and OXPHOS-related protein expression and activated AMPK signaling in the heart, liver, and muscle. These results suggest that BAC induces mitochondrial biogenesis in mice through activating AMPK signaling cascade. BAC may have the potential to be developed as a novel remedy for some diseases associated with mitochondrial dysfunction.
\end{abstract}

Keywords: benzoylaconine; Aconiti Lateralis Radix; traditional Chinese medicine; mitochondrial biogenesis; AMP-activated protein kinase; compound $\mathrm{C}$

Acta Pharmacologica Sinica (2019) 40:658-665; https://doi.org/10.1038/s41401-018-0174-8

\section{INTRODUCTION}

Aconiti Lateralis Radix Praeparata ("Fuzi" in Chinese), the lateral roots of Aconitum Carmichaeli Debx., is a widely used herbal medicine in China. In traditional Chinese medicine (TCM), Fuzi is linked to the Yin-Yang theory and, with the properties of tonifying Yang, increasing heat, and dispersing cold, it has a wide range of applications for treating Yang-deficiency symptoms and Yangdepletion symptoms. In modern research, Fuzi exhibits a wide variety of pharmacological actions, such as effects on the cardiovascular system [1], anti-inflammation activity [2], analgesic action [3], anti-tumor activity [4], and a positive effect on the immune system [5]. Aconitum alkaloids are the primary bioactive compounds in Fuzi, which contribute to its pharmacological efficacy as well as its toxicity, including diester-diterpenoid alkaloids, monoester-diterpenoid alkaloids, and alkylol aminediterpenoid alkaloids $[6,7]$. Aconitine (AC), benzoylaconine (BAC), and aconine are three representative alkaloids. The toxicity of these compounds is markedly decreased with undiminished pharmacological activities when they are exposed to hydrolysis and transformed into BAC-type or aconine-type alkaloids [8]. In detail, BAC is much less toxic than $A C$, with an $L_{50}$ of $1500 \mathrm{mg} / \mathrm{kg}$ in mice $[9,10]$. Some recently published articles proposed a link between Fuzi as a Yang-enhancing agent in TCM and energy metabolism in modern medicine. Fuzi exerts beneficial effects on hepatic energy metabolism by raising the activity of mitochondrial SDH to increase ATP production and by increasing the activity of $\mathrm{Na}^{+}-\mathrm{K}^{+}$-ATPase and $\mathrm{Ca}^{2+}$-ATPase to increase ATP consumption [11]. Another study demonstrated that Fuzi could improve energy metabolism in rats through influencing the metabolism of sugar, lipids, and amino acids. Fuzi was also found to promote the production, storage, and utilization of energy [12]. Moreover, Fuzi ameliorated heart failure in rats by enhancing mitochondrial biogenesis [13], and Fuzi also increased mitochondrial mass [14]. Regarding the individual components of Fuzi, only one article has reported that AC, BAC, and aconine increase intracellular ATP levels and mitochondrial mass in LS174T and Caco-2 cells [15]. However, whether the component alkaloids promote mitochondrial biogenesis and the underlying mechanism remains unclear.

Mitochondria, which are known as the powerhouse of the cell, produce the bulk of cellular ATP through oxidative phosphorylation (OXPHOS). Mitochondrial dysfunction plays a key role in many acute and chronic diseases, ranging from classic mitochondrial diseases that cause energy generation disorders, to a variety of neurological diseases and cancer. Reduced mitochondrial mass,

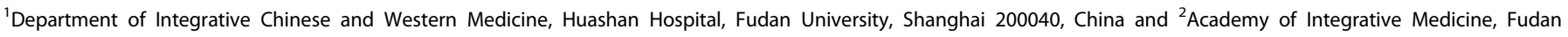
University, Shanghai 200040, China

Correspondence: Jing-cheng Dong (jcdong2004@126.com) or Jian-hua Huang (hjhzyj@aliyun.com)

These authors contributed equally: Xiao-hong Deng and Jing-jing Liu

Received: 5 May 2018 Accepted: 16 September 2018

Published online: 12 October 2018 
decreased mitochondrial function, and impaired mitochondrial biogenesis are common features of aging, neurodegenerative diseases, and diabetes and cardiovascular diseases. Mitochondrial biogenesis is a crucial process for cell viability and survival, and there is growing evidence to support a possible therapeutic role for modulating mitochondrial biogenesis in these diseases [1619]. The most important regulator of mitochondrial biogenesis is peroxisome proliferator-activated receptor- $\gamma$ coactivator-1a (PGC1a) [20, 21]. PGC1a induces mitochondrial biogenesis by activating a group of transcription factors, including nuclear respiratory factor 1 (NRF1) and nuclear respiratory factor 2 (NRF2), which are critical transcriptional regulators of nuclear genes encoding all five electron chain complexes; additionally, PGC1a activates mitochondrial transcription factor A (TFAM) [22, 23]. AMPK, which is upstream of PGC1a, has a critical role in regulating intracellular energy metabolism and is a major regulator of mitochondrial biogenesis in response to chronic energy depletion $[24,25]$. Therefore, AMPK cascade is crucial for mitochondrial biogenesis and ensuring the maintenance of energy homeostasis.

In the present study, we aimed to confirm the effect of Fuzi and its components on mitochondrial biogenesis and to reveal the underlying mechanism in HepG2 cells and in mice. We primarily focused on BAC, owing to its much lower toxicity and higher pharmacological effect on mitochondrial mass. We found that BAC induced mitochondrial biogenesis through activating AMPKPGC1a pathway both in vitro and in vivo.

\section{MATERIALS AND METHODS}

\section{Chemicals and reagents}

The processed Fuzi powder was purchased from Tianjiang Pharmaceutical Co., Ltd. (Jiangyin, Jiangsu, China), dissolved in saline at a concentration of $0.5 \mathrm{~g} / \mathrm{mL}$, and stored at $4{ }^{\circ} \mathrm{C}$. AC, BAC, and aconine (purity $\geq 98 \%$ ) were obtained from Yousi scientific Co., Ltd. (Shanghai, China). AC was dissolved in DMSO, and BAC and aconine were dissolved in saline to a final concentration of 10 $\mathrm{mM}$ and then stored at $-20^{\circ} \mathrm{C}$.

\section{Cell culture and treatment}

The HepG2 cell line was obtained from Shanghai Type Culture Collection of Chinese Academy of Sciences (Shanghai, China). HepG2 cells were cultured in Dulbecco's modified Eagle's medium (DMEM) (Gibco, Waltham, MA, USA) containing 10\% (v/v) fetal bovine serum (FBS) (Gibco) and a 1\% $(v / v)$ penicillin-streptomycin solution (Gibco) at $37^{\circ} \mathrm{C}$ in a humidified atmosphere with $5 \% \mathrm{CO}_{2}$. Fuzi extract $(10 \mathrm{mg} / \mathrm{mL}), \mathrm{AC}(50 \mu \mathrm{M}), \mathrm{BAC}(50 \mu \mathrm{M})$, and aconine $(50$ $\mu \mathrm{M})$ were applied to the cells for $2 \mathrm{~d}$ to investigate their effects on mitochondrial mass. In a separate experiment, BAC at concentrations of $25 \mu \mathrm{M}, 50 \mu \mathrm{M}$, and $75 \mu \mathrm{M}$ or a saline control was applied to the cells. To block AMPK, cells were cultured with compound C ( 2 $\mu \mathrm{M}$, Selleck, Huston, TX, USA) for $2 \mathrm{~h}$ and then co-cultured with $B A C$ for $2 \mathrm{~d}$

Mitochondrial mass

Cells were collected and incubated with $200 \mathrm{nM}$ MitoTracker Green FM (Invitrogen, Waltham, MA, USA) at $37^{\circ} \mathrm{C}$ for $30 \mathrm{~min}$. Mitochondrial mass was measured by fluorescence intensity using flow cytometry (Guava, Millipore, USA).

\section{ATP measurement}

ATP production was examined using an ATP assay kit (Beyotime, Shanghai, China) according to the manufacturer's protocol. Briefly, cells in 6-well microplates were lysed with $100 \mu \mathrm{L}$ of lysis buffer. Next, $100 \mu \mathrm{L}$ of the ATP detection solution was added to a blackflat, clear-bottom 96-well microplate, and incubated for $5 \mathrm{~min}$ at room temperature, and then $20 \mu \mathrm{L}$ of the lysate was added. Chemiluminescence in the reaction mixtures was measured using a microplate reader (Tecan, Switzerland).
Fluorescence microscopy

Cells were fixed with $4 \%$ paraformaldehyde at room temperature for $20 \mathrm{~min}$, washed with PBS, and permeabilized with Triton X-100 for $10 \mathrm{~min}$. The fixed cells were incubated with a rabbit primary antibody against COX4 (Proteintech, Wuhan, China) at room temperature for $90 \mathrm{~min}$, then incubated with an anti-rabbit IgG antibody for $60 \mathrm{~min}$, and finally incubated with DAPI for $15 \mathrm{~min}$. Representative images were acquired using a fluorescence microscope (Zeiss, Germany).

\section{Mitochondrial DNA copy number}

The total genomic DNA of cells and tissues was extracted using TIANamp Genomic DNA kit (Tiangen, Beijing, China). The mtDNA copy number was quantified by real-time PCR using D-loop primers (Human: forward, 5'-ACCCTATGTCGCAGTATCTGTCT-3'; reverse, 5'-CTGTGTGGAAAGTGGCTGTG-3'. Mouse: forward, 5'GGTTCTTACTTCAGGGCCATCA-3'; reverse, 5'- GATTAGACCCGTTA CCATCGAGAT-3'). The following GAPDH primers were used as a nuclear genome control for normalizing the D-loop level (Human: forward, 5'-AAGGTGACAGCAGTCGGTT-3'; reverse, 5' TGTGTGGACTTGGGAGAGG-3'. Mouse: forward, 5'-AAATGGT GAAGGTCGGTG-3'; reverse, 5'-AGGTCAATGAAGGGGTCGTT-3').

\section{Western blotting}

Cells and tissues were lysed in RIPA lysis buffer containing $1 \%$ EDTA (Thermo Fisher Scientific, Waltham, MA, USA) and a protease inhibitor cocktail (Thermo Fisher Scientific). The protein concentrations were determined using a BCA protein assay kit (Thermo Fisher Scientific) according to the manufacturer's protocol. Proteins (30 to $50 \mu \mathrm{g}$ per lane) were separated by $10 \%$ SDSPAGE and electroblotted onto polyvinylidene difluoride membranes (Immobilon-P, Millipore, USA). The membranes were blocked with $5 \%$ BSA-TBS-T, followed by overnight incubation at $4{ }^{\circ} \mathrm{C}$ with the indicated primary antibodies. The following primary antibodies were used: NDUFS1 (1:500, Proteintech), UQCRC1 (1:1000, Proteintech), SDHA (1:1000, Proteintech), COX4 (1:1000, Proteintech), ATP5A1 (1:1000, Proteintech), TFAM (1:500, Proteintech), PGC1a (1:1000, Sigma, St. Louis, MO, USA), NRF1 (1:1000, Cell Signaling Technology, Boston, MA, USA), AMPKa (1:1000, Cell Signaling Technology), and phospho-AMPKa (Thr172) (1:1000, Cell Signaling Technology). $\beta$-actin (1:5000, Cell Signaling Technology) served as the loading control. After immunolabeling, the membranes were washed in TBS-T and incubated with HRPconjugated anti-rabbit, goat, or mouse IgG for $1 \mathrm{~h}$. Immunoreactive bands were detected using an ECL detection kit (Millipore, USA), and a LAS-4000 mini system (Fujifilm Corporation, Tokyo, Japan) was used for visualization.

\section{Gene specific knockdown}

A specific lentiviral system was used to knockdown the expression of PRKAA1 which encodes for AMPKa. We used lenti GV248EGFP shRNA PRKAA1 gaATGCAAAGATAGCTGATTT (GeneChem, Shanghai, China). HepG2 cells were grown in 6-well plates at a density of $2 \times 10^{4}$ cells per well and then transduced for $72 \mathrm{~h}$ with 10 multiplicity of infection (MOI) of lentiviral shRNA directed against PRKAA1 or with a nonsilencing scrambled EGFP negative matched shRNA as control, according to the manufacturer's instructions. Then, the cells were incubated with BAC at 25,50 , and $75 \mu \mathrm{M}$ for $2 \mathrm{~d}$.

\section{Cell proliferation}

The cell proliferation was measured using an EdU (5-ethynyl-2deoxyuridine) imaging kit (Thermo Fisher Scientific) according to the manufacturer's protocol. Treated cells were incubated with a $10-\mu \mathrm{M}$ EdU solution at $37^{\circ} \mathrm{C}$ for $2 \mathrm{~h}$, fixed with $4 \%$ formaldehyde for $15 \mathrm{~min}$, permeabilized with $0.5 \%$ Triton X-100 for $20 \mathrm{~min}$, incubated with a reaction cocktail for $30 \mathrm{~min}$, and then incubated with Hoechst 33342 for $20 \mathrm{~min}$ at room temperature. 
Representative images were acquired using a fluorescence microscope and analyzed using ImageJ software.

Cell viability

Cell viability was examined using a WST-1 assay kit (Beyotime, Shanghai, China) according to the manufacturer's protocol. Briefly, $10 \mu \mathrm{L}$ of WST-1 solution was added to the treated cells in 96-well plates and cultured at $37^{\circ} \mathrm{C}$ for $3 \mathrm{~h}$. The absorbance in the reaction mixtures was measured using a microplate reader (Tecan).

Animal and experimental design

Male Balb/c mice (6-8 weeks old) were obtained from Shanghai JSJ Laboratory Animal Co., Ltd. (Shanghai, China). The mice were housed in an air-conditioned room at $22 \pm 2{ }^{\circ} \mathrm{C}$ with a lighting schedule of $12 \mathrm{~h}$ light and $12 \mathrm{~h}$ dark. Standard chow and tap water were available ad libitum. Mice were acclimatized for 7 days prior to the experiments. All experiments were conducted in accordance with the ethical standards of Experimental Animal Ethics Committee of School of Pharmacy Fudan University (Ethical approval number: 2014-01-HSYY-HJH-01). The mice were randomly divided into the following 2 groups $(n=8)$ : the control group and the BAC group. The control group was treated with vehicle (normal saline, ip), and the BAC group was treated with BAC $(10 \mathrm{mg} / \mathrm{kg}$ per day, ip). The treatments were continued for 7 days.

Oxygen consumption rate

The oxygen consumption rate in the mice was measured using a closed-circuit respirometer according to a previously published protocol $[26,27]$.
Intrascapular temperature

The intrascapular temperature was measured $10 \mathrm{~cm}$ up the backs of the mice using a thermal infrared imager (Flir, Wilsonville, OR, USA) and analyzed using Flir tools.

\section{Statistical analysis}

All results are presented as the mean \pm standard deviation (SD). The statistical significance between two groups was tested using an unpaired $t$-test. Statistical differences were considered significant at $P<0.05$.

\section{RESULTS}

Fuzi extract and its components, AC, BAC, and aconine, increased mitochondrial mass in HepG2 cells

Fuzi extract at $10 \mathrm{mg} / \mathrm{mL}$ and its three primary components, AC, $B A C$, and aconine at a concentration of $50 \mu \mathrm{M}$ all significantly increased MitoTracker Green FM staining (Fig. 1). These results suggest that Fuzi extract and its components promote an increase in mitochondrial mass in HepG2 cells. Thus, any one of these three compounds may represent Fuzi and be used to investigate its underlying mechanisms. However, only BAC was used in the following studies.

BAC increased mitochondrial mass and COX4 expression in HepG2 cells

BAC increased mitochondrial mass at concentrations of $25,50,75$ $\mu \mathrm{M}$ in a dose-dependent manner (Fig. 2a). COX4 is one subunit of the terminal enzyme of the mitochondrial respiratory chain. We found that BAC increased the protein expression of COX4 (Fig. 2b).

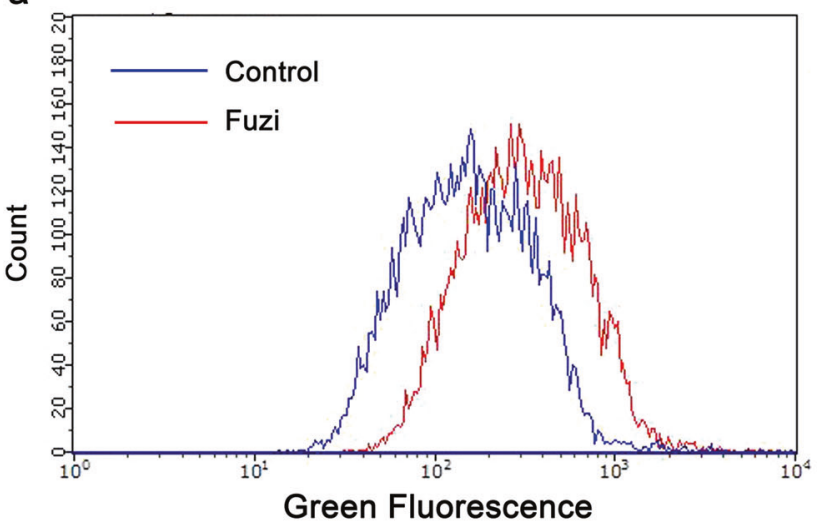

C

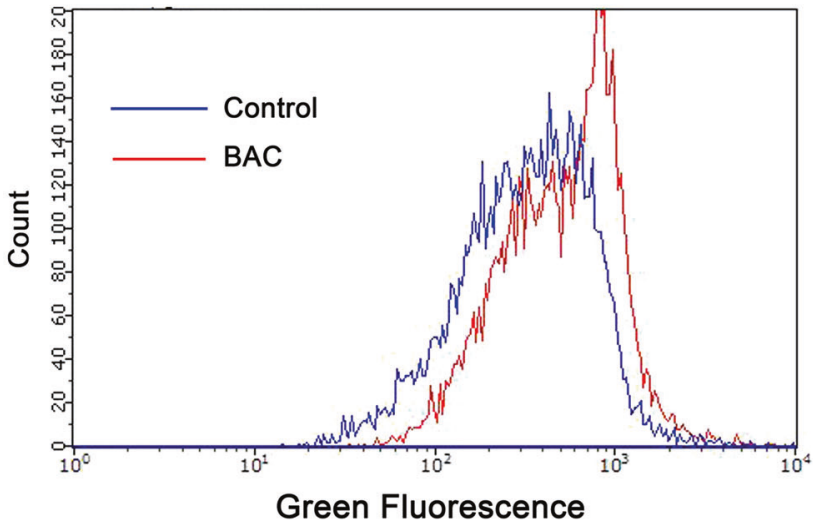

b

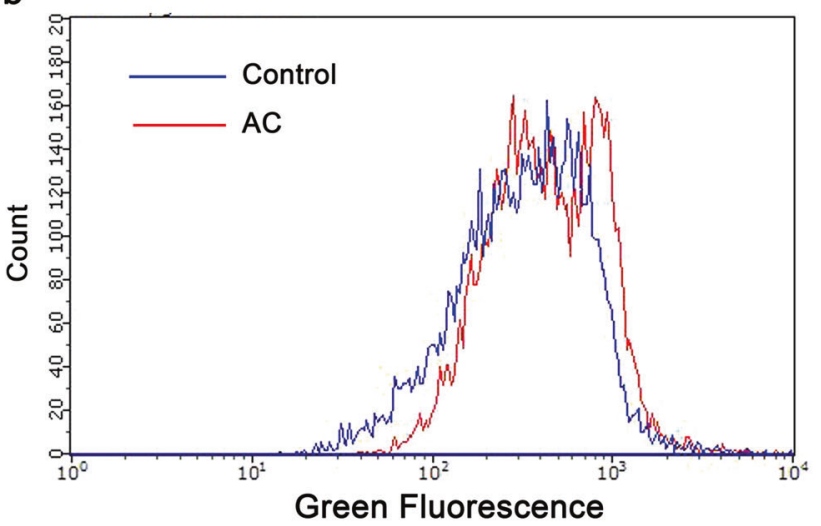

d

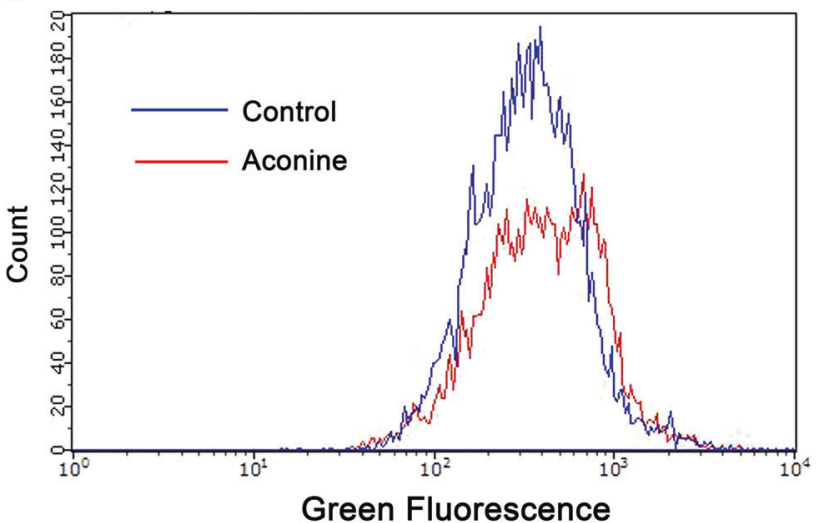

Fig. 1 Fuzi, AC, BAC, and aconine increased mitochondrial mass in HepG2 cells. HepG2 cells were exposed to Fuzi (a), AC (b), BAC (c), and aconine (d). Mitochondrial mass was measured using FACS 
a

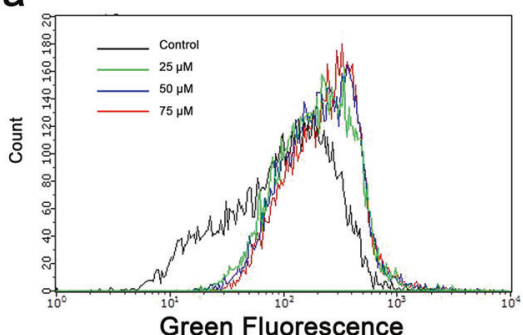

C

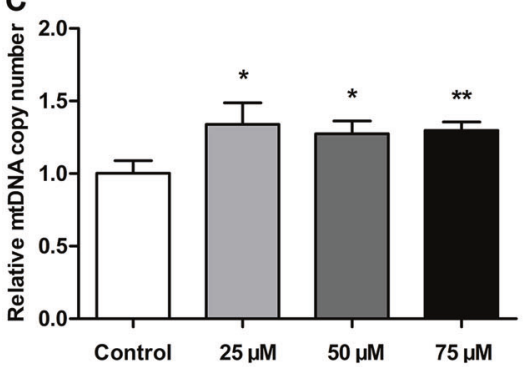

d

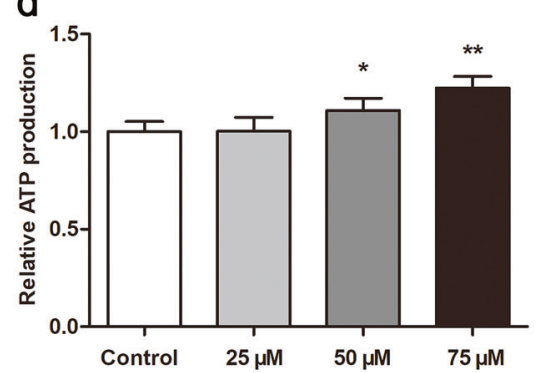

b

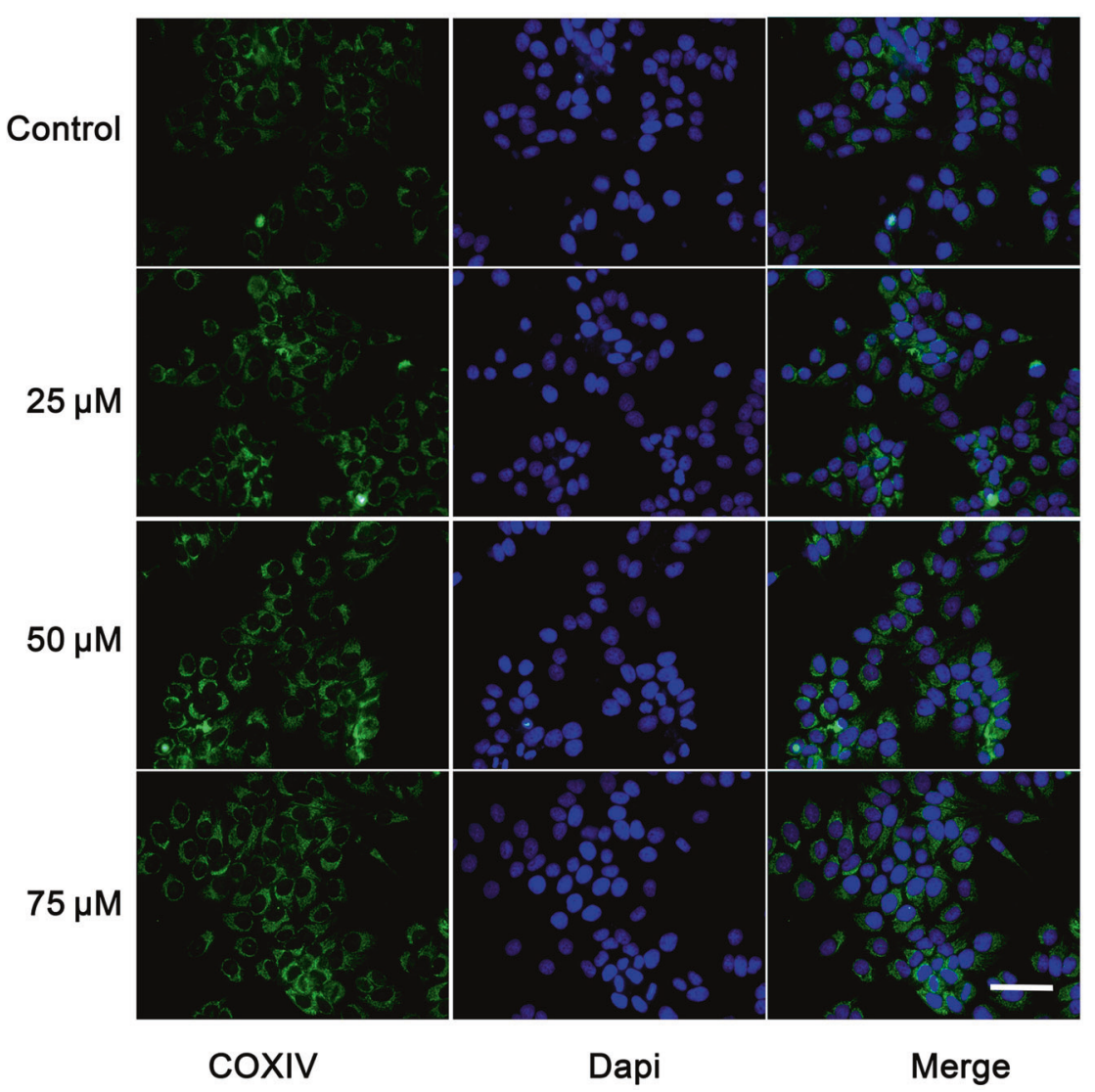

Fig. 2 BAC increased mitochondrial mass and function in HepG2 cells. a HepG2 cells were exposed to BAC at concentrations of 25, 50, and 75

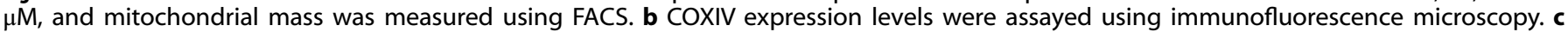
mtDNA copy number was measured by qPCR. d ATP production was measured using an ATP kit. ${ }^{*} P<0.05$, ${ }^{*} P<0.01$. Scale bar, $50 \mu$ m

These results suggest a possible enhancing effect of BAC on mitochondrial biogenesis.

\section{BAC increased mtDNA copy number and ATP production in HepG2 cells}

The mtDNA copy number is an important parameter that directly reflects mitochondrial biogenesis. After exposure to BAC at 25, 50, and $75 \mu \mathrm{M}, \mathrm{mtDNA}$ copy number of HepG2 cells increased by 34 , 27 , and $30 \%$, respectively, compared to the control group (Fig. 2c). ATP production, the most important function of mitochondria, increased by 11 and $22 \%$ after treatment with 50 and $75 \mu \mathrm{M} \mathrm{BAC}$, respectively (Fig. 2d). BAC at $25 \mu \mathrm{M}$ did not increase ATP production (Fig. $2 \mathrm{~d}$ ).

BAC promoted the protein expression of OXPHOS in HepG2 cells Compared with the control group, cells treated with BAC exhibited a notable increase in the protein expression of the OXPHOS subunits, including NDUFS1 (Complex I), SDHA (Complex II), UQCRC1 (Complex III), COX4 (Complex IV), and ATP5A1 (Complex V) (Fig. 3a).

BAC activated AMPK-PGC1a pathway

PGC1a-NRF1-TFAM pathway plays a central role in regulating mitochondrial biogenesis [28]. Therefore, we investigated the effect of BAC on this pathway and found that, indeed, BAC activated the PGC1a-NRF1-TFAM pathway. Compared to the control group, the protein expression levels of PGC1a, NRF1, and TFAM were markedly increased (Fig. 3b). We further examined the effect of BAC on AMPK and its phosphorylation status. It was demonstrated that the levels of AMPKa and p-AMPKa (Thr172) increased as a result of BAC exposure (Fig. 3b).

Blocking AMPK signaling suppressed mitochondrial biogenesis induced by BAC

To confirm that AMPK-PGC1a pathway serves as the master regulator of mitochondrial biogenesis induced by $B A C$, we investigated whether the effect of BAC-induced mitochondrial biogenesis would be suppressed by the antagonist compound $C$ against AMPK or the lentiviral shRNA directed against PRKAA1. Following the inhibition of AMPK activity, the increases in mtDNA copy number induced by BAC were abolished (Fig. 3c, f). Similarly, the protein expression of the OXPHOS subunits decreased significantly (Fig. 3d, g). Moreover, the levels of AMPKa and its phosphorylated counterpart p-AMPKa, as well as PGC1a, NRF1, and TFAM were also reduced (Fig. $3 e, h$ ).

BAC increased cell viability but not cell proliferation in HepG2 cells Treatment with BAC at $6.25-75 \mu \mathrm{M}$ significantly promoted cell viability, as revealed in the WST-1 assay (Fig. 4a). However, BAC did not promote EdU incorporation into cells, suggesting that BAC cannot increase cell proliferation or cell number (Fig. $4 \mathrm{~b}, \mathrm{c}$ ). Taken together, these results indicate that BAC did not promote mitochondrial content via increasing the cell number.

BAC increased the oxygen consumption rate but not the intrascapular temperature in mice

BAC treatment for 3 days increased the oxygen consumption rate by $4 \%$, but this increase was not significant. However, after 
a

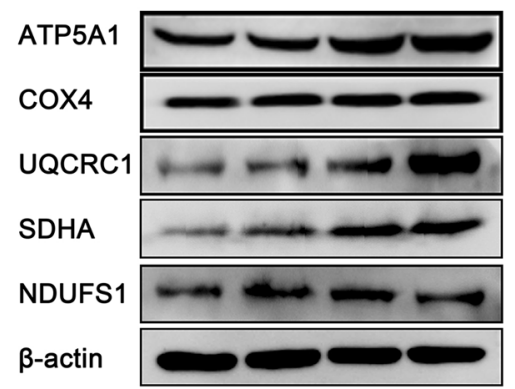

Control $25 \mu \mathrm{M} \quad 50 \mu \mathrm{M} 75 \mu \mathrm{M}$ b

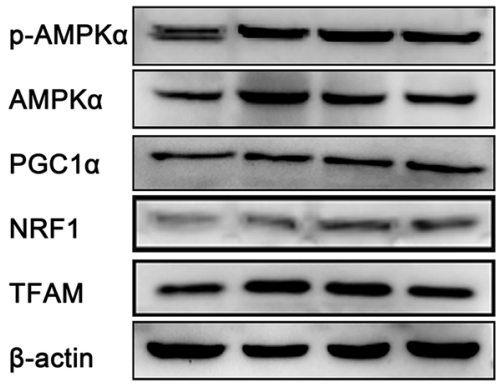

Control $25 \mu \mathrm{M} \quad 50 \mu \mathrm{M} \quad 75 \mu \mathrm{M}$
C

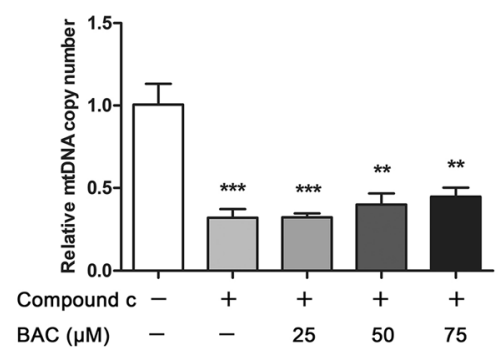

d

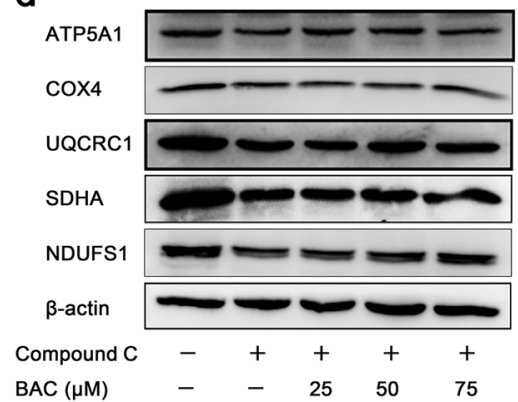

g

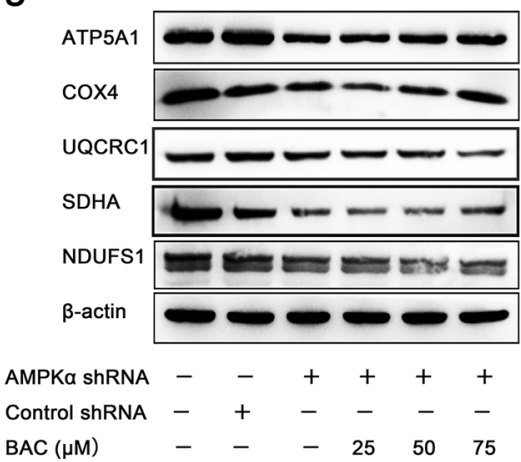

e

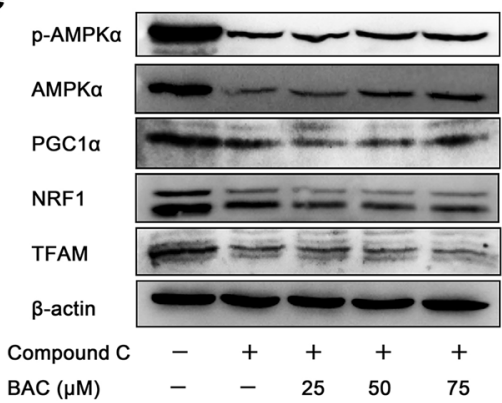

f

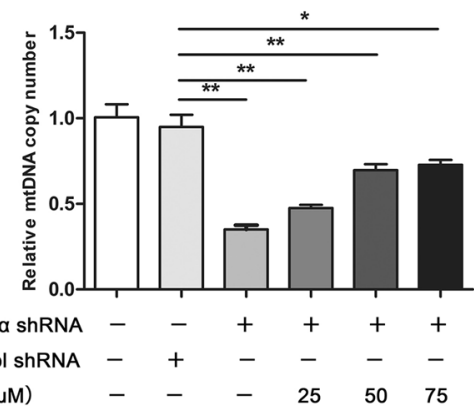

Fig. 3 BAC promoted the expression levels of proteins related to mitochondria biogenesis in HepG2 cells. a The expression levels of OXPHOS

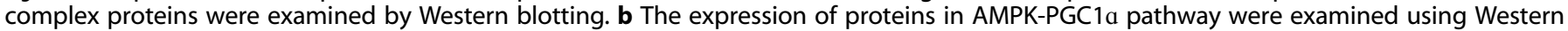

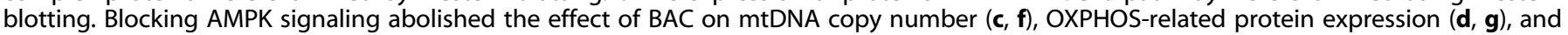
AMPK-PGC1a pathway-related protein expression $(\mathbf{e}, \mathbf{h}) .{ }^{*} P<0.05,{ }^{*} P<0.01,{ }^{* * *} P<0.001$

exposure to $B A C$ for 7 days, the oxygen consumption rate in mice increased by $8 \%$, which was a significant increase (Fig. 5a). Intrascapular temperature reflects the thermogenesis of brown adipose tissue. We measured the temperature of this area and found no significant increase in the intrascapular temperature of the BAC group at the $7^{\text {th }}$ day, compared to the control group (Fig. 5b, c).

BAC induced mitochondrial biogenesis and activated AMPK-

PGC1a pathway in mice

We found that mtDNA copy number of the BAC group in the heart, liver, and muscle increased by 22, 31, and $47 \%$, respectively, compared to the control group (Fig. 6a, d, g). The protein expression of the OXPHOS subunits (NDUFS1, SDHA, UQCRC1, COX4, and ATP5A1) increased as well (Fig. 6b, e, h). Moreover, we confirmed that $B A C$ increased the levels of AMPKa, p-AMPKa, PGC1a, NRF1, and TFAM in mice, suggesting the activation of AMPK- PGC1a pathway in vivo (Fig. 6c, f, i).

\section{DISCUSSION}

Due to the need to respond to diverse metabolic situations, mitochondria should be able to repair or replace their oxidatively damaged parts or increase their mass to maintain function [29]. If mitochondria fail to carry out such repair, then, theoretically, they cannot withstand stress or excessive oxidative injury, and thus they initiate programmed cell death [30]. Indeed, mitochondria do undergo biogenesis [31]. Mitochondrial biogenesis involves replication of the mitochondrial genome followed by division into two daughter mitochondria, a process known as fission. This process has a massive impact on cellular function [32]. Inspired by the knowledge of TCM on the Yang-enhancing effects of Fuzi, two studies that demonstrated that Fuzi promotes mitochondrial biogenesis or increases mitochondrial mass [13, 14] caught our attention.

In the present study, first, we confirmed that Fuzi extract and its three representative components, AC, BAC, and aconine, all increased mitochondrial mass. We focused on BAC in the following studies, given its much lower toxicity and considerable 
a

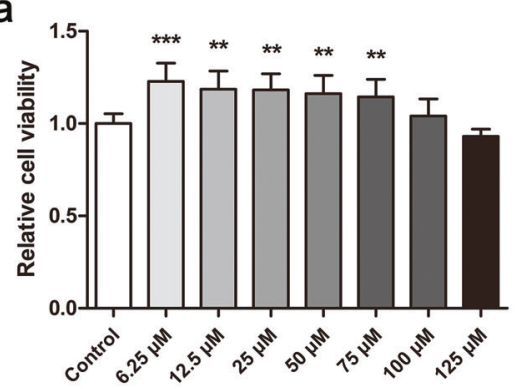

C

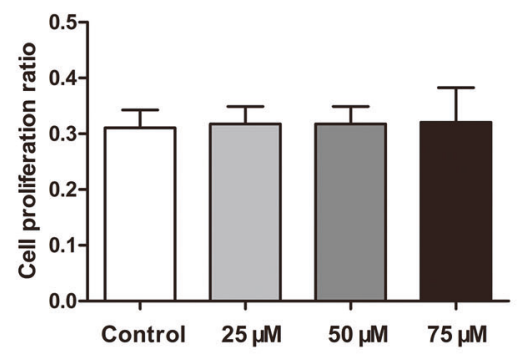

b

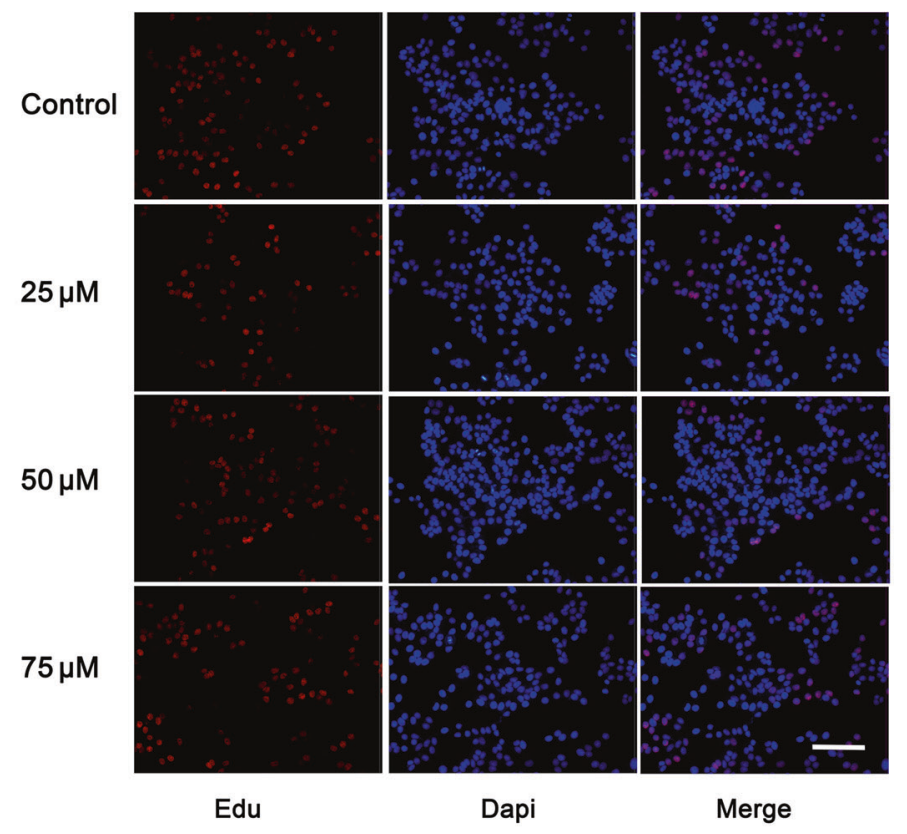

Fig. 4 BAC increased cell viability but not cell proliferation in HepG2 cells. a Cell viability was measured using a WST-1 kit. b Cell proliferation was measured using an Edu imaging kit. c The relative cell proliferation ratio was analyzed using ImageJ software. ${ }^{* *} P<0.01,{ }^{* * *} P<0.001$. Scale bar, $400 \mu \mathrm{m}$

a

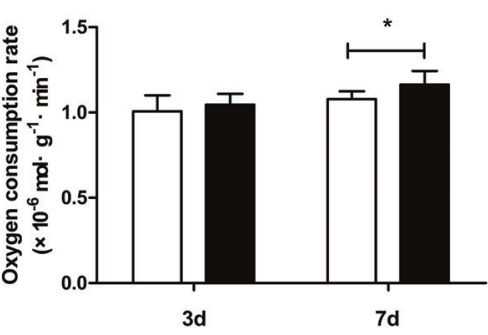

C

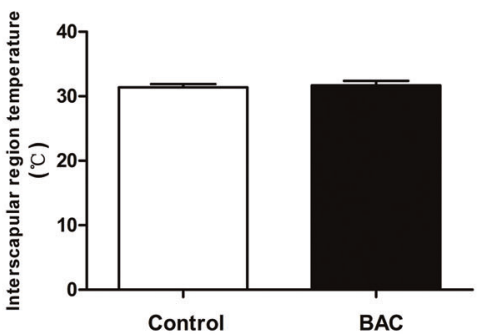

b

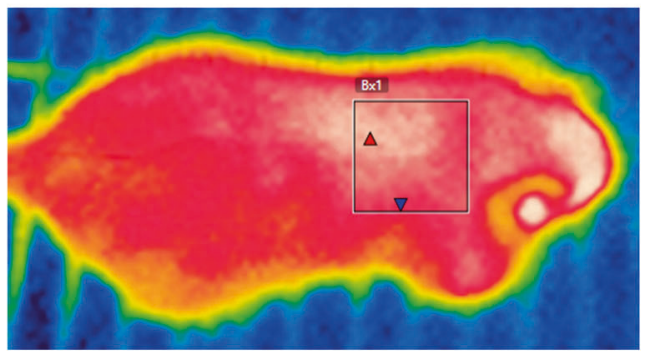

BAC

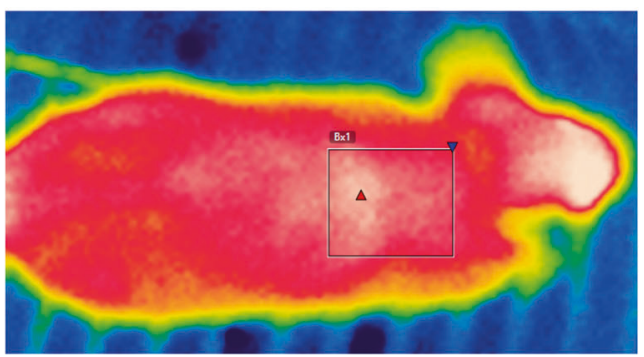

High

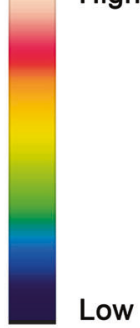

Fig. 5 BAC increased the oxygen consumption rate but not the intrascapular temperature in mice. a The oxygen consumption rate was measured after 7 days of BAC administration. b Intrascapular temperature was measured using a thermal infrared imager. c Average intrascapular temperature was analyzed using the FLIR tolls software. ${ }^{*} P<0.05$

pharmacological activity. We found that BAC increased mitochondrial mass and mtDNA copy number in HepG2 cells. Additionally, cellular ATP production, which is the most important function of mitochondria, increased after exposure to BAC at $50 \mu \mathrm{M}$ and 75 $\mu \mathrm{M}$. The OXPHOS complex plays an essential role in the process of ATP synthesis and mitochondrial function; therefore, we examined the expression levels of OXPHOS. The results revealed that BAC promoted the protein expression of NDUFS1 (complex I), SDHA (complex II), UQCRC1 (complex III), COX4 (complex IV), and ATP5A1 (complex V). Moreover, in mice, BAC increased mtDNA copy number and expression of genes related to mitochondrial biogenesis in three organs with a high metabolic rate-the heart, liver, and skeletal muscle. The oxygen consumption rate is an essential parameter of mitochondrial function. BAC significantly 
a

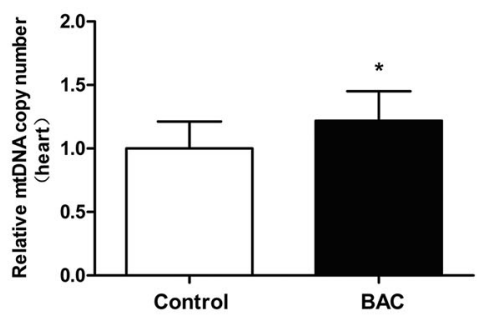

d

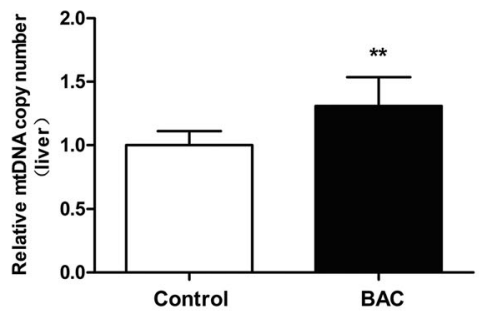

g

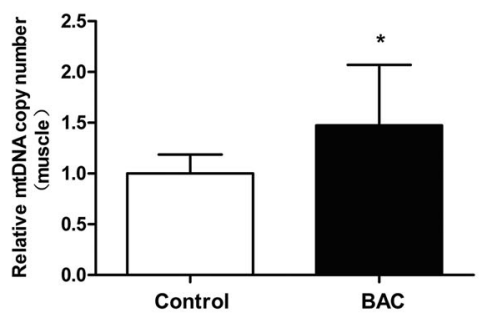

b

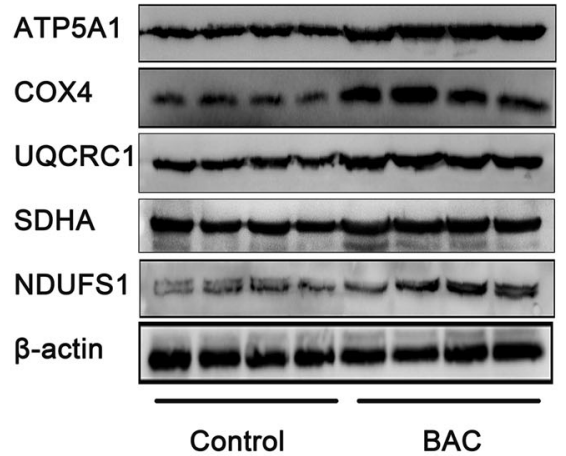

e

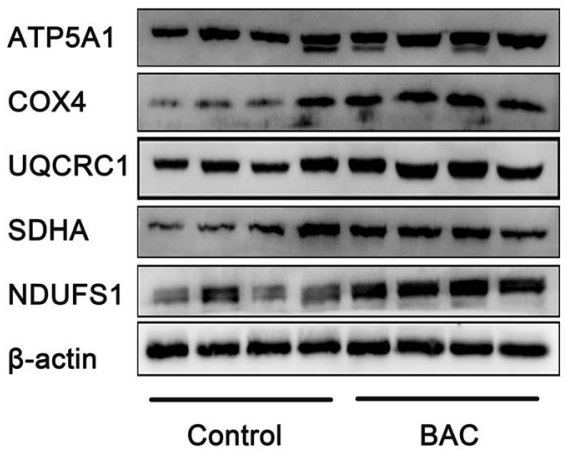

h

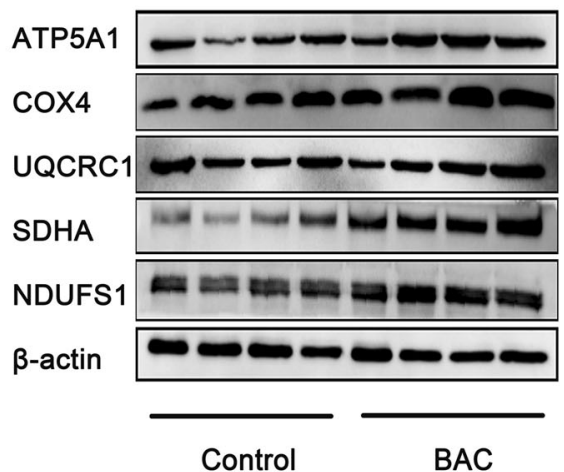

C

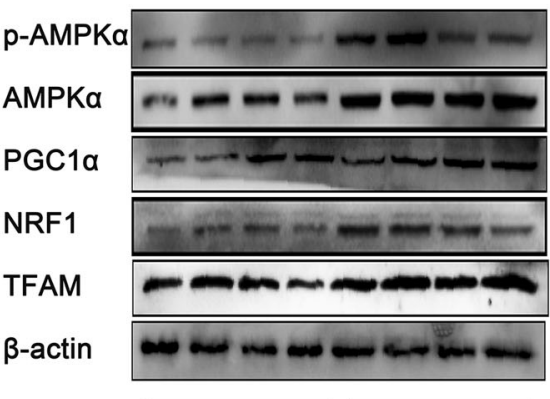

Control

BAC

f

Liver

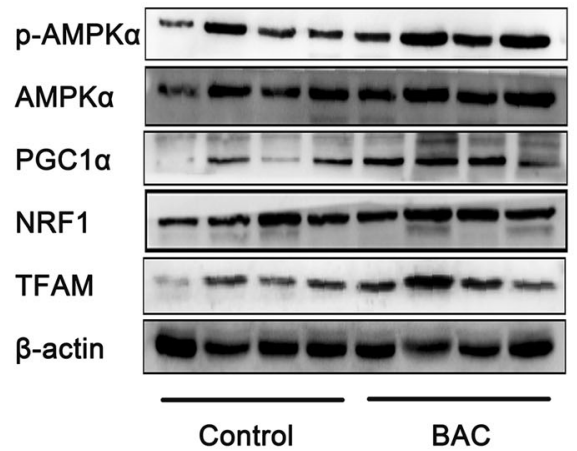

i

Muscle

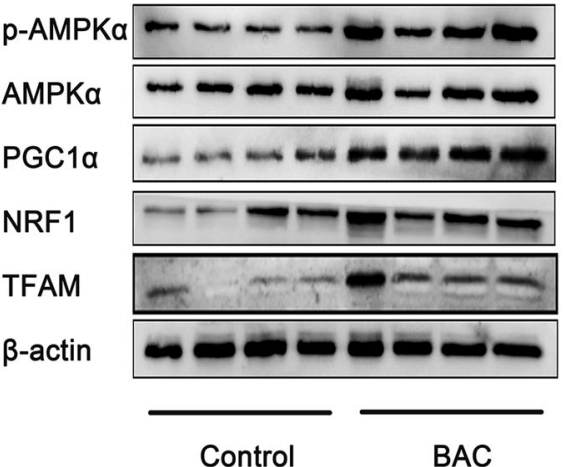

Fig. 6 BAC increased mtDNA copy number and expression levels of proteins related to mitochondrial biogenesis in the heart, liver, and muscle in mice. The mtDNA copy number in heart (a), liver (d), and muscle (g) were measured using q-PCR. The levels of OXPHOS complexrelated proteins in the heart (b), liver (e), and muscle (h) were examined using Western blotting. The expression of AMPK-PGC1a pathwayrelated proteins in the heart (c), liver (f), and muscle (i) was examined using Western blotting

increased the oxygen consumption rate in mice by $8 \%$ after 7 days administration. These findings support the hypothesis that BAC induces mitochondrial biogenesis in vitro and in vivo.

AMPK is a multisubunit enzyme that is recognized as a major regulator of energy metabolism throughout the body. It has been shown that the a subunit Thr 172 is the major AMPK activating site that is phosphorylated by the upstream kinases [33]. Once activated, AMPK regulates its downstream target gene, PGC1a, which is the crucial factor for the activation of the full program of mitochondrial biogenesis [28]. The decreased cellular capacity for mitochondrial biogenesis is associated with reduced levels of PGC1a in various diseases, such as aging, cardiovascular diseases, metabolic diseases, and neurodegenerative diseases [19, 34-36]. PGC1a coordinates the activities of several transcriptional factors involved in mitochondrial biogenesis, including NRF1 and TFAM. In our study, BAC activated AMPK-PGC1 a pathway in HepG2 cells and in mice. To probe the causal link between the effects of $B A C$ and AMPK-PGC1a pathway, the AMPK inhibitor compound $C$ and lentiviral shRNA against PRKAA1 were used. In the presence of these agents, the increased mtDNA copy number and activated expression of proteins related to the OXPHOS and AMPK-PGC1a pathway induced by BAC were markedly reversed.

To the best of our knowledge, the current study is the first to explore the effect of BAC on mitochondrial biogenesis and the underlying mechanism both in vitro and in vivo. Mitochondria play a fundamental role in the survival and function of cardiomyocytes and are critical for the high demand of energy in the myocardium. Mitochondrial biogenesis is severely impaired 
in the human failing heart, as evidenced by the reduction in mtDNA replication and depletion of mtDNA [37]. Garnier et al. found that heart failure induced by aortic banding in rats is associated with the downregulation of all primary transcription factors that promote mitochondrial biogenesis [38]. It has also been reported that aging, metabolic diseases, and neurodegenerative diseases involve mitochondrial dysfunction [16-19]. Stimulation of mitochondrial biogenesis may be a new strategy for the treatment of such disorders. BAC promotes mitochondrial biogenesis, and therefore the potential therapeutic effect of BAC in treating the abovementioned disorders warrants further study.

\section{CONCLUSION}

These in vitro and in vivo findings suggest that BAC increases mitochondrial mass and function through AMPK cascade. Since impaired mitochondrial biogenesis contributes to various diseases, BAC may represent a potential new remedy for treating these disorders.

\section{ACKNOWLEDGEMENTS}

This work was supported by the National Natural Science Foundation of China (grant number 81373791).

\section{AUTHOR CONTRIBUTION}

XHD and JJL contributed equally to this work; XHD and JHH conceived and designed the study; XHD and JJL preformed the experiments and wrote the manuscript; XJS analyzed the data; and JHH and JCD supervised the project. All authors reviewed and approved the manuscript.

\section{ADDITIONAL INFORMATION}

Competing interests: The authors declare no competing interests.

\section{REFERENCES}

1. Zhao D, Wang J, Cui Y, Wu X. Pharmacological effects of Chinese herb aconite (fuzi) on cardiovascular system. J Tradit Chin Med. 2012;32:308-13.

2. Wang Q, Kuang H, Su Y, Sun Y, Feng J, Guo R, et al. Naturally derived antiinflammatory compounds from Chinese medicinal plants. J Ethnopharmacol. 2013;146:9-39.

3. Liou SS, Liu IM, Mei CL, Cheng JT. Comparison of the antinociceptive action of crude Fuzi, the root of Aconitum, and its processed products. J Ethnopharmacol. 2005;99:379-83.

4. Du J, Lu X, Long Z, Zhang Z, Zhu X, Yang $Y$, et al. In vitro and in vivo anticancer activity of aconitine on melanoma cell line B16. Molecules. 2013;18:757-67.

5. Zhao $\mathrm{C}$, Li M, Luo $\mathrm{Y}, \mathrm{Wu} \mathrm{W}$. Isolation and structural characterization of an immunostimulating polysaccharide from fuzi, Aconitum carmichaeli. Carbohydr Res. 2006;341:485-91.

6. Tan G, Liao W, Dong X, Yang G, Zhu Z, Li W, et al. Metabonomic profiles delineate the effect of traditional Chinese medicine Sini decoction on myocardial infarction in rats. PLoS ONE. 2012;7:e34157.

7. Liu J, Li Q, Yin Y, Liu R, Xu H, Bi K. Ultra-fast LC-ESI-MS/MS method for the simultaneous determination of six highly toxic Aconitum alkaloids from Aconiti kusnezoffii radix in rat plasma and its application to a pharmacokinetic study. $J$ Sep Sci. 2014;37:171-8.

8. Singhuber J, Zhu M, Prinz S, Kopp B. Aconitum in traditional Chinese medicine-a valuable drug or an unpredictable risk? J Ethnopharmacol. 2009;126:18-30.

9. Wada K, Nihira M, Hayakawa H, Tomita Y, Hayashida M, Ohno Y. Effects of long term administrations of aconitine on electrocardiogram and tissue concentrations of aconitine and its metabolites in mice. Forensic Sci Int. 2005;148:21-9.

10. Bisset NG. Arrow poisons in China. Part II. Aconitum--botany, chemistry, and pharmacology. J Ethnopharmacol. 1981;4:247-336.

11. Huang L, Peng S, Zhang S, Hu Q, Yu R, Liu H, et al. Hepatic energy metabolism on rats by six traditional Chinese medicine with hot property. Zhongguo Zhong Yao Za Zhi. 2010;35:1470-3.

12. Yu HY, Wang SJ, Teng JL, Ji XM, Wu ZC, Ma QC, et al. Effects of Radix aconiti lateralis preparata and Rhizoma zingiberis on energy metabolism and expression of the genes related to metabolism in rats. Chin J Integr Med. 2012;18:23-9.
13. Lu X, Zhang L, Li P, Wang J, Li R, Huang $Y$, et al. The protective effects of compatibility of Aconiti Lateralis Radix Praeparata and Zingiberis Rhizoma on rats with heart failure by enhancing mitochondrial biogenesis via Sirt1/PGC-1a pathway. Biomed Pharmacother. 2017;92:651-60.

14. Park G, Kim KM, Choi S, Oh DS. Aconitum carmichaelii protects against acetaminophen-induced hepatotoxicity via B-cell lymphoma-2 protein-mediated inhibition of mitochondrial dysfunction. Environ Toxicol Pharmacol. 2016;42:218-25.

15. Wu J, Lin N, Li F, Zhang G, He S, Zhu Y, et al. Induction of P-glycoprotein expression and activity by Aconitum alkaloids: Implication for clinical drug-drug interactions. Sci Rep 2016;6:25343.

16. Wang L, Mascher H, Psilander N, Blomstrand E, Sahlin K. Resistance exercise enhances the molecular signaling of mitochondrial biogenesis induced by endurance exercise in human skeletal muscle. J Appl Physiol. 2011;111: 1335-44.

17. Piao Y, Kim HG, Oh MS, Pak YK. Overexpression of TFAM, NRF-1 and myr-AKT protects the $\operatorname{MPP}(+)$-induced mitochondrial dysfunctions in neuronal cells. Biochim Biophys Acta. 2012;1820:577-85.

18. Zamora M, Pardo $R$, Villena JA. Pharmacological induction of mitochondrial biogenesis as a therapeutic strategy for the treatment of type 2 diabetes. Biochem Pharmacol. 2015;98:16-28.

19. Ren J, Pulakat L, Whaley-Connell A, Sowers JR. Mitochondrial biogenesis in the metabolic syndrome and cardiovascular disease. J Mol Med. 2010;88:993-1001.

20. Wu Z, Puigserver P, Andersson U, Zhang C, Adelmant G, Mootha V, et al. Mechanisms controlling mitochondrial biogenesis and respiration through the thermogenic coactivator PGC-1. Cell. 1999;98:115-24.

21. Irrcher I, Adhihetty PJ, Sheehan T, Joseph A-M, Hood DA. PPARgamma coactivator-1alpha expression during thyroid hormone- and contractile activityinduced mitochondrial adaptations. Am J Physiol Cell Physiol. 2003;284 C1669-77.

22. McGee SL, Hargreaves M. AMPK and transcriptional regulation. Front Biosci. 2008;13:3022-33.

23. Picca A, Lezza AMS. Regulation of mitochondrial biogenesis through TFAMmitochondrial DNA interactions: Useful insights from aging and calorie restriction studies. Mitochondrion. 2015;25:67-75.

24. Jager S, Handschin C, St-Pierre J, Spiegelman BM. AMP-activated protein kinase (AMPK) action in skeletal muscle via direct phosphorylation of PGC-1alpha. Proc Natl Acad Sci USA. 2007;104:12017-22.

25. Hardie DG, Sakamoto K. AMPK: a key sensor of fuel and energy status in skeletal muscle. Physiol (Bethesda). 2006;21:48-60.

26. Liu J, Rao M. A simple method for measuring oxygen consumption in mice. Chin J Pathophysiol. 1996;12:105-7.

27. Stock MJ. An automatic, closed-circuit oxygen consumption apparatus for small animals. J Appl Physiol. 1975;39:849-50.

28. Zhu J, Wang KZQ, Chu CT. After the banquet: mitochondrial biogenesis, mitophagy, and cell survival. Autophagy. 2013;9:1663-76.

29. Nisoli E, Clementi E, Moncada S, Carruba MO. Mitochondrial biogenesis as a cellular signaling framework. Biochem Pharmacol. 2004;67:1-15.

30. Polster BM, Fiskum G. Mitochondrial mechanisms of neural cell apoptosis. J Neurochem. 2004;90:1281-9.

31. Detmer SA, Chan DC. Functions and dysfunctions of mitochondrial dynamics. Nat Rev Mol Cell Biol. 2007;8:870-9.

32. Garnier A, Fortin D, Zoll J, N'Guessan B, Mettauer B, Lampert E, et al. Coordinated changes in mitochondrial function and biogenesis in healthy and diseased human skeletal muscle. FASEB J. 2005;19:43-52.

33. Gowans GJ, Hawley SA, Ross FA, Hardie DG. AMP is a true physiological regulator of AMP-activated protein kinase by both allosteric activation and enhancing net phosphorylation. Cell Metab. 2013;18:556-66.

34. Sheng B, Wang X, Su B, Lee H, Casadesus G, Perry G, et al. Impaired mitochondrial biogenesis contributes to mitochondrial dysfunction in Alzheimer's disease. J Neurochem. 2012;120:419-29.

35. Joseph AM, Adhihetty PJ, Buford TW, Wohlgemuth SE, Lees HA, Nguyen LM-D, et al. The impact of aging on mitochondrial function and biogenesis pathways in skeletal muscle of sedentary high- and low-functioning elderly individuals. Aging Cell. 2012;11:801-9.

36. Lai C-Q, Tucker KL, Parnell LD, Adiconis X, Garcia-Bailo B, Griffith J, et al. PPARGC1A variation associated with DNA damage, diabetes, and cardiovascular diseases: the Boston Puerto Rican Health Study. Diabetes. 2008;57:809-16.

37. Karamanlidis G, Nascimben L, Couper GS, Shekar PS, Del Monte F, Tian R. Defective DNA replication impairs mitochondrial biogenesis in human failing hearts. Circ Res. 2010;106:1541-8.

38. Garnier A, Fortin D, Deloménie C, Momken I, Veksler V, Ventura-Clapier R. Depressed mitochondrial transcription factors and oxidative capacity in rat failing cardiac and skeletal muscles. J Physiol. 2003;551:491-501. 